\title{
Facoemulsificação sob anestesia tópica realizada por residentes do terceiro ano de Oftalmologia
}

\author{
Phacoemulsification under topical anesthesia \\ performed by third year Ophthalmology residents
}

Marina Soares Viegas Moura Rezende', Rafael Roedel Sperb², Édson Iramina², Simone de Biagi Souza', Luiz Eduardo Feliciano Ribeiro ${ }^{3}$, Omar Dib ${ }^{4}$

\section{Resumo}

Objetivos: Avaliar a eficácia e a segurança da transição da cirurgia de facoemulsificação sob anestesia peribulbar para tópica realizadas por residentes do terceiro ano. Métodos: Os prontuários dos pacientes submetidos à facoemulsificação no período de agosto de 2004 a janeiro de 2005 foram analisados. Os pacientes foram operados sob anestesia tópica (grupo I) e peribulbar (grupo II). Foram obtidos dados referentes a sexo, idade, acuidade visual com estenopeico e complicações. Foram excluídos pacientes com baixa acuidade visual não devido à catarata. Resultados: Cento e cinqüenta e um (151) olhos foram avaliados; 70 olhos no grupo I e 81 no grupo II. A acuidade visual pósoperatória com estenopeico aos seis meses foi superior a logMAR $+0,30$ em $80,7 \%$ e a média foi de log MAR +0.10 em ambos os grupos. As análises das complicações intraoperatórias entre os grupos I e II mostrou diferença estatisticamente significante $(p=0,039)$.Conclusão: Nossos dados sugerem que a transição para cirurgia de facoemulsificação sob anestesia tópica realizadas por residentes de terceiro ano é eficaz e segura.

Descritores: Facoemulsificação; Anestesia local; Anestesia/métodos; Corpo clínico hospitalar

\footnotetext{
Médicas do Instituto de Oftalmologia Tadeu Cvintal - São Paulo (SP), Brasil;

${ }^{2}$ Residentes de Terceiro Ano do Instituto de Oftalmologia Tadeu Cvintal - São Paulo (SP), Brasil;

Médico Preceptor do Instituto de Oftalmologia Tadeu Cvintal - São Paulo (SP), Brasil;

${ }^{4}$ Chefe do Serviço de Catarata do Instituto de Oftalmologia Tadeu Cvintal - São Paulo (SP), Brasil.
} 


\section{INTRODUÇÃO}

A técnica anestésica de escolha para facectomia evoluiu com o tempo. Desde introduzida, a anestesia local administrada por injeção peribulbar ou retrobulbar foram os métodos de eleição. Embora sejam eficazes em produzir anestesia e acinesia, estas formas de anestesia local não estão desprovidas de complicações oculares e sistêmicas que ocorrem raramente, mas que podem ser severas. Estas incluem estrabismo, hemorragia retrobulbar, perfuração do globo ocular, e problemas cardiopulmonares ${ }^{(1.3)}$.

A anestesia tópica para cirurgia de catarata foi possível desde que Koller introduziu a cocaína tópica em 1884. Embora em 1995 menos de 10\% dos oftalmologistas americanos usassem anestesia tópica, a Sociedade Americana de Catarata e Cirurgia Refrativa, em relação à técnica anestésica empregada, em levantamento de 2001 mostra que o bloqueio peribulbar foi usado por $19 \%$, o retrobulbar com bloqueio facial por $11 \%$ e a anestesia tópica por $56 \%$ dos pesquisados. A anestesia tópica apresenta as vantagens da rápida reabilitação e boa aceitação do paciente enquanto também reduz os riscos associados com anestesia peribulbar ${ }^{(4.5)}$.

A segurança e eficácia da anestesia tópica comparada à técnica peribulbar têm sido favoráveis, quando realizada por cirurgiões experientes (1) , e a transição da anestesia local para a tópica por estes cirurgiões tem sido avaliada. Porém, raros estudos têm sido publicados demonstrando a taxa de sucesso da anestesia tópica realizada por cirurgiões em treinamento. Apesar da eliminação das complicações da anestesia peribulbar ser um motivo importante, a menor habilidade técnica dos residentes supõe ser a transição para a anestesia tópica potencialmente mais complicada o que poderia levar a um número maior de complicações e baixa acuidade visual.

Este estudo examinou e comparou o resultado da facoemulsificação realizada por cirurgiões residentes usando anestesia tópica e peribulbar.

\section{Métodos}

Os prontuários médicos dos pacientes submetidos à facoemulsificação no período de 31 de Julho de 2004 a 31 de Janeiro de 2005, foram acessados. As cirurgias foram realizadas por dois residentes do terceiro ano da Catarata orientados por médicos assistentes, do Instituto de Oftalmologia Tadeu Cvintal, no Hospital Dom Antônio de Alvarenga, em São Paulo.

Nos casos feitos com anestesia tópica (grupo I) foi utilizada cloridrato de proximetacaína $0,5 \%$, uma gota de colírio anestésico de 5 em 5 minutos, 15 minutos antes da cirurgia.

Nos casos em que foi realizada anestesia peribulbar (grupo II), foi utilizada uma mistura de Bupivacaína $0,75 \%$ (2ml) e lidocaína 2\% (2,5ml) sem epinefrina injetada no espaço peribulbar utilizando uma agulha de $25 \times 7 \mathrm{~mm}$.

A técnica utilizada nas cirurgias foi relativamente uniforme. Os residentes iniciaram com incisão tipo "clear-cornea" aproximadamente às 10 horas. A capsulorrexis foi realizada, e o núcleo emulsificado, utilizando a técnica de "stop and chop". A lente intra-ocular utilizada foi acrílica, hidrofóbica e dobrável, inserida no saco capsular. Em alguns casos, a lente foi introduzida no sulco ciliar por questões técnicas, como rotura posterior de saco capsular.

Todos os prontuários foram avaliados. Os dados pré-operatórios foram: idade, sexo, olho operado, acuidade visual sem correção (com estenopeico) e condições oculares prévias limitantes da acuidade visual. Os dados intra-operatórios para cada caso foram: o residente cirurgião, o cirurgião assistente, a data da cirurgia, o tipo de anestesia realizada, o tipo e poder de lente intra-ocular, o número do caso do residente, e as complicações intra-operatórias.

As informações pós-operatórias incluíram a acuidade visual no sétimo e trigésimo dia com estenopeico. As complicações durante o seguimento também foram avaliadas.

Os pacientes foram excluídos da análise quando havia baixa acuidade visual não devido à catarata diagnostica no pré-operatório. Nos casos de patologias oculares diagnosticadas no pós-operatório, os pacientes foram incluídos na análise dos resultados e suas patologias relatadas.

\section{Resultados}

Dos 151 olhos identificados, todos (100\%) tiveram a informação necessária e foram incluídos na análise.

A média de idade observada foi de 68 anos, variando de 41 a 87 anos. Houve predomínio do sexo feminino com 82 casos $(54,3 \%)$ (Tabela 1$)$.

A acuidade visual pré-operatória com uso de estenopeico variou de movimentos de mão a LogMar +0.2 parcial.

No total, 70 casos $(46,3 \%)$ foram realizados com anestesia tópica e $81(53,6 \%)$ com anestesia peribulbar.

Ocorreram complicações intra-operatórias em 
sete casos: ruptura de cápsula posterior ( 3 casos, todos em pacientes realizados com anestesia peribulbar), perda vítrea (2 casos realizados com anestesia peribulbar), $1 / 4$ de núcleo no vítreo ( 1 casos realizado com anestesia peribulbar) e quebra da háptica da LIO (1 casos realizado com anestesia peribulbar) (Tabela 2).

As complicações pós-operatórias incluíram edema de córnea, opacidade de cápsula posterior, edema macular cistóide, puntata e córnea nebulosa como mostrados ( Tabela 3).

Cento e vinte e dois (122) casos $(80,7 \%)$ obtiveram acuidade visual (AV) com estenopeico de logMAR +0.3 ou melhor. Quando casos de patologias oculares diagnosticadas no pós-operatório (7 casos com as seguintes patologias: DMRI, retinopatia diabética, cicatriz de coriorretinite, atrofia do EPR, buraco macular) foram eliminados, $82,1 \%$ adquiriram uma $\mathrm{AV}$ com estenopeico de $\log$ MAR +0.3 ou melhor. A mediana entre as acuidades visuais aos seis meses de cirurgia para o grupo I foi igual a 1,000, semelhante a encontrada para o grupo II. A média entre as acuidades visuais foi de $\log \mathrm{MAR}+0.10$ para o grupo I e $\log \mathrm{MAR}+0.10$ para o grupo II. O teste de Mann-Whitney comparou os resultados das acuidades visuais, aos seis meses, de ambos grupos e revelou $p=0,928$.

Foram observados 15 pacientes com acuidade visual com estenopeico aos 6 meses menor que logMAR $+0,5$ (Tabela 4).

\section{Dıscussão}

Embora a segurança e eficácia da anestesia tópica para cirurgia de facoemulsificação feita por cirurgiões experientes tenha sido bem documentada, só encontramos um estudo sobre facoemulsificação realizada sob anestesia tópica por residentes ${ }^{(5)}$. Para avaliar a segurança da anestesia tópica quando realizada por residentes, foram comparados os dados deste trabalho e também de outros que comparam os dois métodos sendo que com cirurgiões experientes ${ }^{(1.3)}$.

Os dois grupos apresentavam características demográficas semelhantes. Os casos do grupo I foram realizados após os residentes terem feito aproxidamente 75 casos com peribulbar previamente.

As complicações intra-operatórias ocorreram todas no grupo II, o teste exato de Fisher revelou $p=0,039$ (Tabela 2). A perda vítrea foi de 1,9\%, um número favorável quando comparado a taxas da literatura de casos feitos por residentes $(4,1 \%)^{(6)}$.

Não houve diferença estatisticamente significante entre os dois grupos $(p=0,282)$ no que se
Tabela 1

Aspectos demográficos

\begin{tabular}{lccc}
\hline Aspectos demográficos & Tópica & Peribulbar & Total \\
\hline Total de casos & 70 & 81 & 151 \\
Média de idade & 68 & 70 & 69 \\
Sexo masculino & 32 & 37 & 69 \\
Sexo feminino & 38 & 44 & 82 \\
\hline
\end{tabular}

Tabela 2

Complicações intra-operatórias

\begin{tabular}{lccc}
\hline Complicações & Tópica & Peribulbar & Total \\
\hline Háptica quebrada & 0 & 1 & 1 \\
Ruptura de cápsula posterior & 0 & 3 & 3 \\
Perda vítrea & 0 & 2 & 2 \\
$1 / 4$ de núcleo no vítreo & 0 & 1 & 1 \\
\hline
\end{tabular}

Teste exato de fisher $p=0,039$

Tabela 3

\section{Complicações pós-operatórias}

\begin{tabular}{lccc}
\hline Complicações & Tópica & Peribulbar & Total \\
\hline Edema de córnea persistente & 0 & 1 & 1 \\
Opacidade de cápsula posterior & 1 & 0 & 1 \\
Edema macular cistóide & 3 & 2 & 5 \\
Descentração da LIO & 1 & 0 & 1 \\
\hline
\end{tabular}

Teste exato de Fisher $p=0,282$

Tabela 4

Razões para acuidade visual
menor que 0.3 (logmar+0,5)

\begin{tabular}{lccc}
\hline & \multicolumn{3}{c}{ Tópica Peribulbar } \\
\hline Edema de córnea persistente & 0 & 2 & 2 \\
Opacidade de cápsula posterior & 1 & 0 & 1 \\
Edema macular cistóide & 2 & 2 & 4 \\
Condições prévias * & 2 & 5 & 7 \\
Desconhecidas & 0 & 1 & 1 \\
\hline
\end{tabular}

*condições diagnosticadas pós-cirurgia (DMRI, retinopatia diabética, cicatriz de cório retinite, atrofia do EPR, buraco macular) 
refere a complicações pós-operatórias (Tabela 3).

Nossos resultados indicam que para cirurgiões com algum treinamento prévio em facoemulsificação com anestesia peribulbar, a transição para anestesia tópica pode evoluir com resultados de acuidade visual semelhante àqueles feitos com peribulbar, sem aumento das taxas de complicações pós-operatórias.

Em nosso estudo não foi avaliada a opinião dos pacientes em relação à técnica anestésica empregada, informação importante e destacada por outros trabalhos. Crandall et al. opinam que a segurança da cirurgia de catarata sob anestesia tópica aumenta com a experiência do cirurgião e o preparo do paciente, apesar de maior sensibilidade pós-operatória encontrada nos casos de anestesia tópica, os autores em 13 casos onde houve cirurgia bilateral em uso das duas técnicas discutidas obtiveram o favoritismo de oito pacientes pela anestesia tópica contra cinco pela peribulbar ${ }^{(4)}$. Helena Kallio et al. encontraram a preferência, entre pacientes submetidos a diferentes técnicas, pela anestesia tópica com sedação de propofol ${ }^{(7)}$.

Um estudo prospectivo randomizado com maior número de casos, que considere o conforto do paciente e do cirurgião com cada técnica, possibilitaria uma melhor comparação entre os dois grupos. Entretanto, nosso estudo mostra que os residentes podem iniciar, após algum tempo de treinamento com bloqueio peribulbar, facoemulsificação com segurança, utilizando anestesia tópica desde que com adequada supervisão.

\section{Abstract:}

Purpose: To evaluate the outcome and safety of the transition from phacoemulsification performed by third year residents using peribulbar anesthesia to topical anesthesia. Methods: Cases performed by third year residents from august 2004 to february 2005 were analyzed. Patients were operated under peribulbar (group
I) and topical anesthesia (group II). Variables analyzed included patients age, sex, visual acuity under pinhole and complications. Patients with low visual acuity other than cataract were excluded from analysis. Results: A total of 151 eyes were analyzed; 70 eyes in group I and 81 in group II. The visual acuity with pinhole as better than $\log M A R+0,30$ in $80,7 \%$ and the average visual acuity after six months was log MAR+0.10 for both groups. The complication rate diference between both groups was statistically significant $(p=0,039)$. Conclusion: Our data suggests that transition to topical anesthesia performed by third year residents is safe with similar outcomes.

Keywords: Phacoemulsification; Anesthesia, local; Anesthesia/methods, Medical staff, hospital

\section{ReferênCias}

1. Patel BC, Burns TA, Crandall A, Shomaker ST, Pace NL, van Eerd A, Clinch T. A comparison of topical and retrobulbar anesthesia for cataract surgery. Ophthalmology. 1996; 103(8):1196-203.

2. Roman S, Auclin F, Ullern M. Topical versus peribulbar anesthesia in cataract surgery. J Cataract Refract Surg. 1996; 22(8):1121-4.

3. Zehetmayer M, Radax U, Skorpik C, Menapace R, Schemper $\mathrm{M}$, Weghaupt H, Scholz U. Topical versus peribulbar anesthesia in clear corneal cataract surgery. J Cataract Refract Surg. 1996; 22(4):480-4.

4. Crandall AS, Zabriskie NA, Patel BC, Burns TA, Mamalis N, Malmquist-Carter LA, Yee R. A comparison of patient comfort during cataract surgery with topical anesthesia versus topical anesthesia and intracameral lidocaine. Ophthalmology. 1999; 106(1):60-6.

5. Leaming DV. Practice styles and preferences of ASCRS members - 2001 survey. J Cataract Refract Surg. 2002; 28(9):1681-8.

6. Randleman JB, Srivastava SK, Aaron MM. Phacoemulsification with topical anesthesia performed by resident surgeons. J Cataract Refract Surg. 2004; 30(1): 149-54.

7. Kallio H, Uusitalo RJ, Maunuksela EL. Topical anesthesia with or without propofol sedation versus retrobulbar/peribulbar anesthesia for cataract extraction: prospective randomized trial. J Cataract Refract Surg. 2001; 27(9):1372-9. 